\title{
Pesticide Toxicity Profile: Synthetic Pyrethroid Pesticides 1
}

\author{
Frederick M. Fishel $^{2}$ \\ This document provides a general overview of \\ human toxicity, provides a listing of laboratory \\ animal and wildlife toxicities and a cross reference of \\ chemical and common names with their trade names \\ of many synthetic pyrethroid pesticides registered for \\ use in Florida.
}

\section{General}

Pyrethrins were originally derived from East African chrysanthemum flowers and shown to have insecticidal activity. In a natural environment, they were chemically unstable and broke down rapidly upon exposure to air and sunlight. Beginning in the 1970s, synthetic pyrethroids came into the market for agricultural purposes as they were synthesized from petroleum derivatives. They are also widely used as home and garden insecticides along with uses on pets and livestock, mosquito control, treatment of transport vehicles, and for treatment of ectoparasitic disease. Their desirable features are providing a quick knockdown of insects at low rates, relatively low mammalian toxicity and improved stability in outdoor environments, which has increased their marketability in agriculture. They are effective against a wide range of insect and mite pests and may be mixed with other pesticides for a broad spectrum of pest control. Formulations that are commercially available include aerosols, dips, emulsifiable concentrates, wettable powders, granules, and concentrates for ultra low volume applications targeting mosquitoes. Pyrethroids may be mixed with piperonyl butoxide, a synergist, which enhances the effect of the active ingredient. Their mode of action is interference with transmission of nerve impulses.

\section{Toxicity}

Pyrethroids are one of the least acutely toxic insecticides to mammals because they are quickly deactivated by metabolic processes. However, rats fed high doses $(1,000 \mathrm{mg} / \mathrm{kg}$ of body weight) showed liver damage (Hayes, 1982). Toxicity by inhalation and dermal absorption is low. Sensitization sometimes occurs in some individuals after a single exposure which causes either an asthmatic condition or a skin rash or inflammation. After the initial exposure to the sensitizing agent, the sensitized

1. This document is PI-54, one of a series of the Agronomy Department, Florida Cooperative Extension Service, Institute of Food and Agricultural Sciences, University of Florida. Published July, 2005. Visit the EDIS Web Site at http://edis.ifas.ufl.edu.

2. Frederick M. Fishel, Associate Professor, Agronomy Department, and Director, Pesticide Information Office; Florida Cooperative Extension Service, Institute of Food and Agricultural Sciences, University of Florida, Gainesville, FL 32611.

The use of trade names in this publication is solely for the purpose of providing specific information. UF/IFAS does not guarantee or warranty the products named, and references to them in this publication does not signify our approval to the exclusion of other products of suitable composition. Use pesticides safely. Read and follow directions on the manufacturer's label.

The Institute of Food and Agricultural Sciences (IFAS) is an Equal Opportunity Institution authorized to provide research, educational information and other services only to individuals and institutions that function with non-discrimination with respect to race, creed, color, religion, age, disability, sex, sexual orientation, marital status, national origin, political opinions or affiliations. U.S. Department of Agriculture, Cooperative Extension Service, University of Florida, IFAS, Florida A. \& M. University Cooperative Extension Program, and Boards of County Commissioners Cooperating. Larry Arrington, Dean 
individual responds to a dose smaller than the initial dose. Symptoms are more common with exposure to the pyrethroids whose structures include

cyano-groups. Sensations are described as stinging, burning, itching, and tingling, progressing to numbness, with the face most commonly affected. Persons treated with permethrin for lice or flea infestations sometimes experience itching and burning at the site of application, but this is more of a reaction to the effects of the parasites themselves. Due to the inclusion of certain solvents, some formulations of fluvalinate are corrosive to the eyes. Scientists have no data from work-related, accidental poisonings, or epidemiological studies that indicate whether or not pyrethrins are likely to cause cancer in humans. There were no birth defects in pups of rabbits exposed to pyrethrins (Vettorazzi, 1979); however, rat pups born to rats fed very high doses of pyrethrins for three weeks prior to mating were of low body weights (Hayes, 1982). Pyrethrins are highly toxic to fish and tadpoles. They affect their skin touch receptors and balance organs (Tomlin, 1994). Mammalian toxicities for pyrethroid pesticides registered in Florida are shown in Table 1. Table 2 lists the toxicities to wildlife by the common name of the pyrethroid pesticide. Table 3 provides a cross listing of many of the trade names that these products are registered and sold by in Florida.

\section{Additional Information}

Crop Protection Handbook. 2005. vol. 91. Willoughby, Ohio: Meister Publishing Co. http://www.meisterpro.com/mpn.

Hayes, W.J. 1982. Pesticides studied in man. Baltimore: Williams and Wilkins.

Nesheim, O.N., F.M. Fishel and M.A. Mossler. 2005. Toxicity of Pesticides UF/IFAS EDIS Document PI-13. (http://edis.ifas.ufl.edu/PI008)

Reigart, J.R. and J.R. Roberts. 1999. Recognition and management of pesticide poisonings, $5^{\text {th }}$ edition. United States Environmental Protection Agency Publication EPA-735-R-98-003.

Seyler, L.A., et.al. 1994. Extension toxicology network (EXTOXNET). Cornell University and Michigan State University. http://extoxnet.orst.edu/index.html. Visited July 2005.

Tomlin, C. (Ed.). 1994. A World Compendium. The Pesticide Manual. Incorporating the agrochemicals handbook. (10 ${ }^{\text {th }}$ ed.). Bungay, Suffolk, U.K.: Crop Protection Publications.

Vettorazzi, G. 1979. International regulatory aspects for pesticide chemicals. Toxicity Profiles (vol. 1), Boca Raton, FL: CRC Press, Inc. 
Table 1. Pyrethroid pesticide mammalian toxicities ( $\mathrm{mg} / \mathrm{kg}$ of body weight).

\begin{tabular}{|l|c|c||}
\hline \hline Common name & Rat oral LD & Rabbit dermal LD $_{\mathbf{5 0}}$ \\
\hline Allethrin & 860 & 11,332 \\
\hline Bifenthrin & 375 & $>2,000$ \\
\hline Cyfluthrin & $869-1271$ & $>5,000$ (rat) \\
\hline Cyhalothrin & 79 & 632 (rat) \\
\hline Cypermethrin & 250 & $>2,000$ \\
\hline Deltamethrin & $31-139$ (female) & $>2,000$ \\
\hline Esfenvalerate & 451 & 2,500 \\
\hline Fenpropathrin & $70.6-164$ & $>2,000$ \\
\hline Fluvalinate & $261-282$ & $>20,000$ \\
\hline Permethrin & $430-4,000$ & $>2,000$ \\
\hline Resmethrin & $1,244->2,500$ & $>2,500$ \\
\hline Tefluthrin & 969 & $>2,000$ (rat) \\
\hline Tetramethrin & $>5,000$ & $>2,000$ \\
\hline Tralomethrin & 284 & $>2,000$ \\
\hline \hline
\end{tabular}

Table 2. Pyrethroid pesticide wildlife toxicity ranges.

\begin{tabular}{|c|c|c|c|}
\hline Common name & Bird acute oral LD ${ }_{50}(\mathrm{mg} / \mathrm{kg})^{\star}$ & Fish LC $_{50}(p p m)^{\star \star}$ & Bee $L D_{50}{ }^{\dagger}$ \\
\hline Allethrin & PNT & HT & HT \\
\hline Bifenthrin & ST - PNT & HT & $\mathrm{HT}$ \\
\hline Cyfluthrin & PNT & VHT & $\mathrm{HT}$ \\
\hline Cyhalothrin & PNT & $\mathrm{HT}$ & $\mathrm{HT}$ \\
\hline Cypermethrin & PNT & VHT & $\mathrm{HT}$ \\
\hline Deltamethrin & PNT & $\mathrm{HT}$ & HT \\
\hline Esfenvalerate & PNT & VHT & HT \\
\hline Fenpropathrin & ST & VHT & $\mathrm{HT}$ \\
\hline Fluvalinate & PNT & VHT & MT \\
\hline Permethrin & PNT & VHT & $\mathrm{HT}$ \\
\hline Resmethrin & PNT & VHT & $\mathrm{HT}$ \\
\hline Tefluthrin & ST - PNT & VHT & $\mathrm{HT}$ \\
\hline Tetramethrin & PNT & $\mathrm{HT}$ & --- \\
\hline Tralomethrin & --- & VHT & $\mathrm{HT}$ \\
\hline \multicolumn{4}{|c|}{$\begin{array}{l}{ }^{*} \text { Bird } L_{50} \text { : Practically nontoxic }(\mathrm{PNT})=>2,000 ; \text { slightly toxic }(\mathrm{ST})=501-2,000 ; \text { moderately toxic }(\mathrm{MT})= \\
51-500 ; \text { highly toxic }(\mathrm{HT})=10-50 \text {; very highly toxic }(\mathrm{VHT})=<10 . \\
{ }^{* \star} \mathrm{Fish}_{\mathrm{LC}} \text { : PNT }=>100 ; \mathrm{ST}=10-100 ; \mathrm{MT}=1-10 ; \mathrm{HT}=0.1-1 ; \mathrm{VHT}=<0.1 . \\
{ }^{\dagger} \text { Bee: } \mathrm{HT}=\text { highly toxic (kills upon contact as well as residues); } \mathrm{MT}=\text { moderately toxic (kills if applied over } \\
\text { bees); PNT = relatively nontoxic (relatively few precautions necessary). }\end{array}$} \\
\hline
\end{tabular}


Table 3. Cross reference list of common, trade and chemical names of pyrethroid insecticides.

\begin{tabular}{|c|c|c|}
\hline $\begin{array}{l}\text { Common } \\
\text { name* }^{*}\end{array}$ & Trade names ${ }^{\star *}$ & Chemical name \\
\hline Allethrin & $\begin{array}{l}\text { Many household } \\
\text { products }\end{array}$ & $\begin{array}{l}\text { (RS)-3-allyl-2-methyl-4-oxycyclopent-2-enyl (1RS)-cis-trans } \\
\text { chrysanthemate }\end{array}$ \\
\hline Bifenthrin & Capture $\AA$, Talstar ${ }^{\circledR}$ & $\begin{array}{l}\text { [1-alpha,3-alpha-(Z)]-(+)-(2 methyl[1,1'-biphenyl]-3yl) methyl } \\
\text { 3-(2,chloro-3,3,3-trifluoro-1-propenyl)-2,2-dimethylcyclopropanecarboxylate }\end{array}$ \\
\hline Cyfluthrin & Baythroid $\AA$, Tame $\AA$ & $\begin{array}{l}\text { Cyano(4-fluoro-3-phenoxyphenyl)methyl } \\
\text { 3-(2,2-dichloro-ethenyl)-2,2-dimethylcyclopropanecarboxylate }\end{array}$ \\
\hline Cyhalothrin & $\begin{array}{l}\text { Karate } \AA, \text { Warrior } \AA \text {, } \\
\text { Demand } \AA \text {, Scimitar } \AA\end{array}$ & $\begin{array}{l}\text { alpha-cyano-3-phenoxybenzyl } \\
\text { 3-(2-chloro-3,3,3-trifluoroprop-1-enyl)-2,2-dimethylcyclopropanecarboxylate }\end{array}$ \\
\hline Cypermethrin & $\begin{array}{l}\text { Ammo®, Fury® } \\
\text { Mustang® }\end{array}$ & $\begin{array}{l}( \pm) \text {-alpha-cyano-3-phenoxybenzyl } \\
( \pm) \text {-cis,trans-3-(2,2-dichlorovinyl)-2,2-dimethycyclopropanecarboxylate }\end{array}$ \\
\hline Deltamethrin & $\begin{array}{l}\text { Decis } \AA \text {, DeltaGard } \AA \text {, } \\
\text { Demand } \AA\end{array}$ & $\begin{array}{l}\text { (S)-cyano(3-phenoxybenzyl) } \\
\text { (1R,3R)-3-(2,2-dibromovinyl)-2,2-dimethylcyclopropanecarboxylate }\end{array}$ \\
\hline Esfenvalerate & Asana® & $\begin{array}{l}\text { (S)-cyano(3-phenoxyphenyl)methyl } \\
\text { (S)-4-chloro-alpha-(1-methylethyl)-benzeneacetate }\end{array}$ \\
\hline Fenpropathrin & Danitol $\AA$, Tame $\AA$ & $\begin{array}{l}\text { RS-alpha-cyano-3-phenoxybenzyl } \\
\text { 2,2,3,3-tetramethylcyclopropanecarboxylate }\end{array}$ \\
\hline Fluvalinate & Mavrik®, Zoecon® & $\begin{array}{l}\text { Á-RS,2R)-fluvalinate [(RS)-alpha-cyano-3-phenoxybenzyl } \\
(\mathrm{R})-2 \text {-[2-chloro-4-(trifluoromethyl)anilino]-3-methyl-butanoate] }\end{array}$ \\
\hline Permethrin & Ambush $\AA$, Pounce $\AA$ & $\begin{array}{l}\text { (3-phenoxyphenyl)methyl } \\
\text { ( } \pm \text {-cis,trans-3-(2,2-dichloroethenyl)-2,2-dimethylcyclopropanecarboxylate }\end{array}$ \\
\hline Resmethrin & $\begin{array}{l}\text { Many household } \\
\text { products }\end{array}$ & $\begin{array}{l}\text { ([5-(phenylmethyl)-3-furanyl]methyl } \\
\text { 2,2-dimethyl-3-(2-methyl-1-proenyl)cyclopropanecarboxylate) }\end{array}$ \\
\hline Tefluthrin & Force $\AA$ & $\begin{array}{l}\text { 2,3,5,6-tetrafluoro-4-methylbenzyl (Z)-(1 RS, } \\
\text { 3RS)-3-(2-chloro-3,3,3-trifluoroprop-1-ethyl) } \\
\text {-2,2-dimethylcyclopropanecarboxylate }\end{array}$ \\
\hline Tetramethrin & $\begin{array}{l}\text { Many household } \\
\text { products }\end{array}$ & 3,4,5,6-tetrahydrophthalimidomethyl (1RS)-cis,trans-chrysanthemate \\
\hline Tralomethrin & Scout $(\AA$ & $\begin{array}{l}(1 \mathrm{R}, 3 \mathrm{~S}) 3\left[\left(1^{\prime}, 2^{\prime}, 2^{\prime}, 2^{\prime},- \text { tetrabromoethyl)]-2,2-dimethylcyclopropanecarboxylic }\right.\right. \\
\text { acid (S)-alpha-cyano-3-phenoxybenzyl ester }\end{array}$ \\
\hline
\end{tabular}

